\title{
Advanced Practices on Detection and Classification of Diabetic Retinopathy from Fundus Images
}

\author{
Ashima Gambhir
}

\begin{abstract}
Medical Image processing is extremely trendy research area now days in this category only digital images are diagnosed. Diabetic patients can have eye disease recognized as Diabetic Retinopathy. Diabetic Retinopathy(DR) is the general origin of sightlessness in middle and advanced mature group people. Therefore it is essential to identify Diabetic Retinopathy (DR) at the early stages and give suitable treatment according to the severity. It is a worldwide illness that affects equivalent to 80 percent of patients those are suffering with diabetes problem with minimum 10 years history. In Diabetic Retinopathy, these are the some components like hemorrhages, Microanurysms, hard and soft exudates. Currently a large number of computerized algorithms have been made to retrieve precise retinal blood vessel from fundus images. This paper presents the categorization the different stages of the Diabetic Retinopathy and different achievable retinal blood vessels segmentation algorithms and their examination are discussed.
\end{abstract}

KEYWORDS-Diabetic Retinopathy; Hemorrhages; Microanurysms; Exudates; NPDR; PDR.

\section{INTRODUCTION}

Diabetic Retinopathy (DR) is spoil to the retina of the eye due to rise of sugar level in blood, which can ultimately heading towards sightlessness. The retina is inner layer of eye which is very sensitive. As diabetes passage in the patient, the disease gradually affects the circulatory organism of the body including the retina of the eye and produces a result of harm to the tiny blood vessels of the retina; decline the vision of the patient leading to diabetic retinopathy[6]. These small blood vessels will leak fluid and blood on the retina in forms features like microanurysms, hemorrhages, hard and soft exudates, cotton wool spots.

Manuscript received May 2, 2020

Ashima Gambhir, Amity School of Engineering \& Technology, Amity University, Haryana, Gurgaon, India (email: ashima.ashugambhir@gmail.com)
There are huge numbers of people, who are affliction from Diabetic retinopathy(DR) that leads to blurring of vision or even blindness. Hence, it is required to detect and extraction the retinal blood vessels for Diabetic Retinopathy detection and diagnosis, which prevents earlier vision loss in diabetic patient. The proper detection and early treatment of diabetes is used to save much amount because implications of the late treatment and improper or poor treatment are much more costly. Fundoscopy is a technique in which internal structure of retina is examined to detect the problem. Therefore, it plays an significant task in diabetic retinopathy(DR) recognition and monitoring. Extraction of blood vessels is major task for detection of diabetic Retinopathy (DR). Digital image processing is applied to field of medical diagnosis for reducing the time and stress which is undergone by ophthalmologist for diagnosis and treatment of DR.

\section{CATEGORIZATION OF DIABETIC RETINOPATHY}

Diabetic Retinopathy (DR) is categorize into different types founded on sternness. Diabetic Retinopathy classified into two different stages named as proliferative diabetic retinopathy (PDR) and nonproliferative diabetic retinopathy (NPDR).

\section{A. Nonproliferative Diabetic Retinopathy(NPDR)}

This type of DR is classified into three category named Mild NPDR, Moderate NPDR, Severe NPDR[13].

- Mild Non proliferative Diabetic retinopathy is the initial stage of DR. It is categorized by the existence of dots and red mark haemorrhages and Microanurysms in the retina of an eye. Haemorrhages are positioned in the focal point layer of eye retina. Haemorrhages mean unusual blood flow from the vessels in the retina of the eye. Microanurysms are the areas of puffiness of the insignificant blood vessels in the retina of the eye caused by declining of their composition.

- Moderate Non proliferative Diabetic retinopathy is the second stage of Diabetic retinopathy. During this phase, a number of the minute blood vessels in the retina of the eye may in fact become jammed due to diminish in the supply of oxygen and nutrients to the particular areas of the eye retina.

- Severe Non proliferative Diabetic retinopathy is a major number of tiny retinal blood vessels in the retina becoming blocked, resulting in additional areas of the retina. Lack of 
sufficient oxygen and nutrients supply to the blood vessels of the retina marks in a situation called Retinal Ischemia.

\section{B. Proliferative Diabetic Retinopathy(PDR)}

Proliferative Retinopathy is a final stage of DR in which abnormal new blood vessel start growing to facilitate carries a important possibility of vision loss. The rate of growing of these new blood vessels is very high. Due to growing of abnormal retinal blood vessels, eye retina responds to a be short of of oxygen by attempting to recompense for the compact flow. The abnormal blood vessels are exceedingly weak and lean to split effortlessly. It may origin the retina to crumple or disconnect. The more rigorous vision failure is seen in the PDR than NPDR as it is responsible for affecting both central and peripheral vision. Fig.1, explains the types of Diabetic Retinopathy(DR).

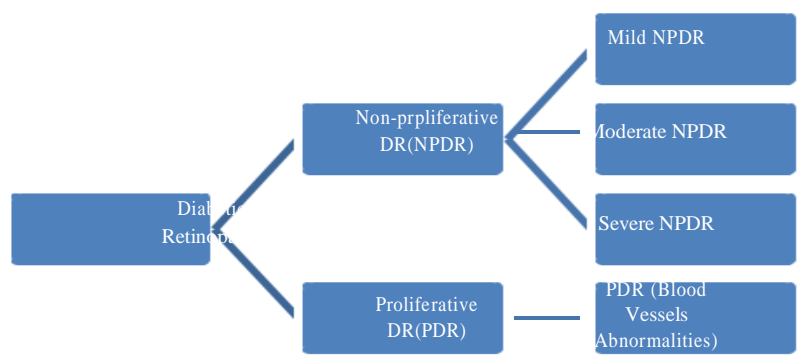

Fig 1: Categorization of Diabetic Retinopathy

Figure 2 explain the different stages of the diabetic retinopathy based on severity [13].
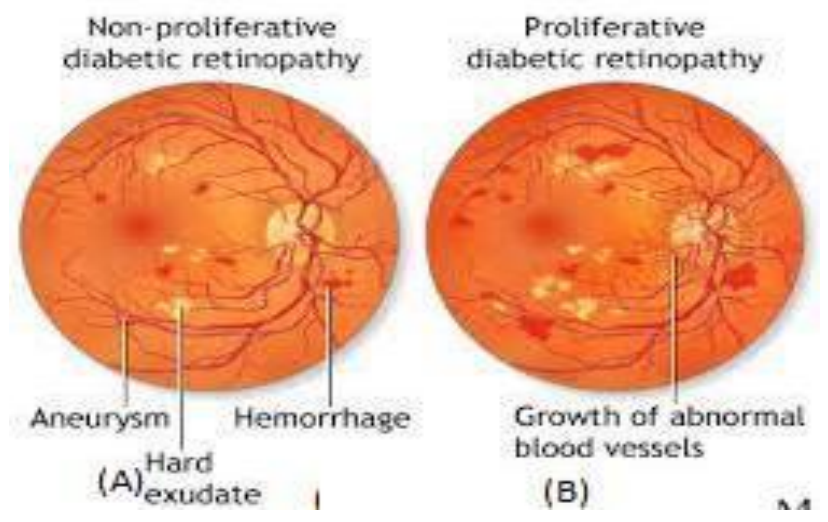

Fig 2: Stages of Diabetic Retinopathy (A) Signs of NPDR (B) Signs of PDR

\section{PUBLICLY AVAILABLE DATABASES}

Fundus Camera is a device is used to retrieve the images of retina of the eye and these images are known as Retinal Fundus Images (RFI)[11]. Fundus camera takes the images of internal surface of retina, blood vessels, optic disc and macula. Some Online databases are available for the research on medical retinal or fundus images. The main purpose of the available databases is to check the potency, screening of Diabetic Retinopathy and compare the results with current techniques[13]. Table 1 gives an overview of online available fundus images databases including DRIVE, STARE, Messidor, Retinopathy Online Challenge (ROC), Image Ret, HEI-MED.

\section{LITERUTRE SURVEY}

Many approaches and algorithms are proposed by various researches for detection of different stages of DR. In this section an overview of the existing work, their research gaps and future scope is discussed.

- G.R. Hemalakshmi, N.B. Prakash, , M. Stella[7] In this paper, the finding of optic disk is made by means of $\mathrm{K}$ means clustering techniques, blood vessel segmentation by means of Kirsh's template edge detection algorithm and exudates detection by means of intensity computation, threshloding and feature extraction. The exudates are classified with the help of SVM classifier and were able to distinguish between four different types of grading level or stages with an average accuracy of $94.17 \%$.

- D. Siva Sundhara Raja, S. Vasuki[4] Have proposed the computer aided automatic detection and segmentation for retinal blood vessels. In this manuscript, a methodology is used which automatically detected and segmented the retinal blood vessel by eliminating OD(Optic Disk) regions from the retinal image in order to increase the segmentation accuracy level for blood vessel segmentation. The authors achieved the average vessel segmentation accuracy of 98.08\% in DRIVE Database and 95.94\% in STARE Dataset respectively, with their corresponding ground truth images.

- JayakumarLachure[3] Detect the Hard, soft exudates and Microanurysms for regular detection of DR. SVM and KNN classifier are used. Exudates are detected by Morphological operations such as 
Table 1: Available Databases for Fundus Images

\begin{tabular}{|c|c|c|c|c|}
\hline Database & $\begin{array}{c}\text { Image } \\
\text { possession }\end{array}$ & Description & Resolution & Uses \\
\hline DRIVE & $\begin{array}{l}\text { 3 CCD Camera } \\
\text { with } 45 \text {-fold } \\
\text { view }\end{array}$ & $\begin{array}{l}20 \text { color testing images and } 20 \\
\text { color training images with } \\
\text { extracted blood vessels }\end{array}$ & $768 \times 584$ & $\begin{array}{c}\text { Detection of abnormal blood vessels, exudates, } \\
\text { hemorrhages, Microanurysms }\end{array}$ \\
\hline STARE & $\begin{array}{l}50 \text { fundus } \\
\text { camera with } 35- \\
\text { fold view }\end{array}$ & $\begin{array}{l}400 \text { images with masked and } \\
\text { extracted blood vessels }\end{array}$ & $605 \times 700$ & $\begin{array}{l}\text { Exudates, Microanurysms, Hemorrhages and abnormal } \\
\text { blood vessels detection }\end{array}$ \\
\hline HET-MED & $\begin{array}{c}\text { ZeissVisucam } \\
\text { PRO fundus } \\
\text { camera at } 45 \text { fold } \\
\text { view } \\
\end{array}$ & $\begin{array}{c}\text { Total } 169 \text { color fundus images out } \\
\text { of which } 115 \text { are abnormal and } 54 \\
\text { images are healthy }\end{array}$ & $2196 \times 1958$ & Exudates Detection \\
\hline
\end{tabular}

- Dilation, final and erosion operators are used Number of MAs(Microanurysms) is counted and grade is calculated. Grades and hyper plane representation for multiclassification are used to measure the performance of the system. SVM is better than KNN because SVM provides specificity, sensitivity is more than KNN.

- M.Raghupathy, R.ManjulaSri and K.M.M.Rao[5] This paper described the different stages of Diabetic Retinopathy(DR), NPDR and PDR. Input character Red, Green and Blue(RGB) color of perimeter and areas of retinal blood vessels are extracted from retinal image using mathematical morphological technique and algorithm is implemented in Lab View.

- M. Ponnibala et al [1]A new method for extracting blood vessels from color fundus image based on feature classification It reduce time for examining retinal images. Reprocessing method and in segmentation technique, matched filter and modified local entropy thresholding operation are performed. CLAHE technique which operates on small data regions. True(positive) and False(Negative) are used as parameters for performance measurement.ELM is better than SVM because it provides a specificity, sensitivity accuracy more than SVM.ELM provides easy computation and successful screening method for diagnosing PDR.

- MalavikaBhaskaranand[2]Automated tool for screening DR patients and its extension forestimating the MA turnover via longitudinal analysis.DR screening tool automatically determines colour retinal fundus images. MA turnover estimation tool aligns retinal images from multiple encounters of patients and estimates MA turnover rates. This tools are potential biomarker for DR risk.

\section{COMPARATIVE ANALYSIS}

For detection and classification of DR disease, some authors discussed about the techniques and algorithms for preprocessing of fundus images[12]. Table 2 shows the relative examination for recognition and categorization of Diabetic Retinopathy(DR) using different techniques. 


\section{Advanced Practices on Detection and Classification of Diabetic Retinopathy from Fundus Images}

Table 2: Techniques and Algorithms used for Detection and Categorization of Diabetic Retinopathy(Dr)

\begin{tabular}{|c|c|c|c|}
\hline Technique and algorithm & Database & Classifier & Conclusion and Results \\
\hline $\begin{array}{lr}\mathrm{K} & \text { mean Clustering Technique } \\
\text { and } & \text { Kirsh's template edge } \\
\end{array}$ & e-Optha-Ex Database & SVM Classifier & $\begin{array}{l}\text { Distinguish the four types of Diabetic } \\
\text { Retinopathy with an average accuracy of }\end{array}$ \\
\hline detection algorithm & & & $94.17 \%$. \\
\hline $\begin{array}{l}\text { Binary morphological operations } \\
\text { are used over threshold image }\end{array}$ & DRIVE and STARE Database & - & $\begin{array}{l}\text { Accuracy of } 98.08 \% \text { in DRIVE Database and } \\
95.94 \% \text { in STARE Dataset. }\end{array}$ \\
\hline $\begin{array}{l}\text { Morphological operations } \\
\text { dilation, final and attrition } \\
\text { operators are } \\
\text { used }\end{array}$ & Own Database & $\begin{array}{l}\text { SVM and K-nearest } \\
\text { neighbours Classifier }\end{array}$ & $\begin{array}{l}\text { SVM is superior than KNN because SVM } \\
\text { provides specificity } 100 \% \text { and sensitivity is } \\
\text { more than } 90 \%\end{array}$ \\
\hline $\begin{array}{lr}\text { Mathematical morphological } \\
\text { Techniques \& algorithm is } \\
\text { implemented in Lab View. }\end{array}$ & Messidor Dataset & - & Accuracy of $85 \%$ in Messidor dataset. \\
\hline $\begin{array}{l}\text { Pre-processing method, } \\
\text { segmentation technique, matched } \\
\text { filter and modified local entropy } \\
\text { thresholding operation, CLAHE }\end{array}$ & $\begin{array}{l}\text { Lotus Eye Care Hospital } \\
\text { Coimbatore Database }\end{array}$ & ELM Classifier & $\begin{array}{l}\text { ELM is better than SVM because it provides } \\
\text { a specificity, sensitivity accuracy of } 96.66 \% \text {, } \\
100 \% \text { and } 97.5, \% 100 \% \text { and } 94.11 \%, 95 \%\end{array}$ \\
\hline DR screening tool & EyePACS Dataset & $\begin{array}{l}\text { Learning Ensemble } \\
\text { classifier }\end{array}$ & $\begin{array}{l}\text { The DR screening tool achieves } 90 \% \\
\text { compassion at } 63.2 \% \text { specificity. }\end{array}$ \\
\hline
\end{tabular}

\section{PROPOSED METHODOLOGY}

Several approaches and algorithms have been already proposed by various researchers for detection and classification of different stages of DR. In this, I proposed a different technique to improve the segmentation by extracting thin blood vessel without noise from retinal fundus images and evaluate the perimeter and area of blood vessels to find the hard, soft exudates, Hemorrhages and Microanurysms in the retina of the eye. Fig. 3, depicts the flow chart of proposed methodology.

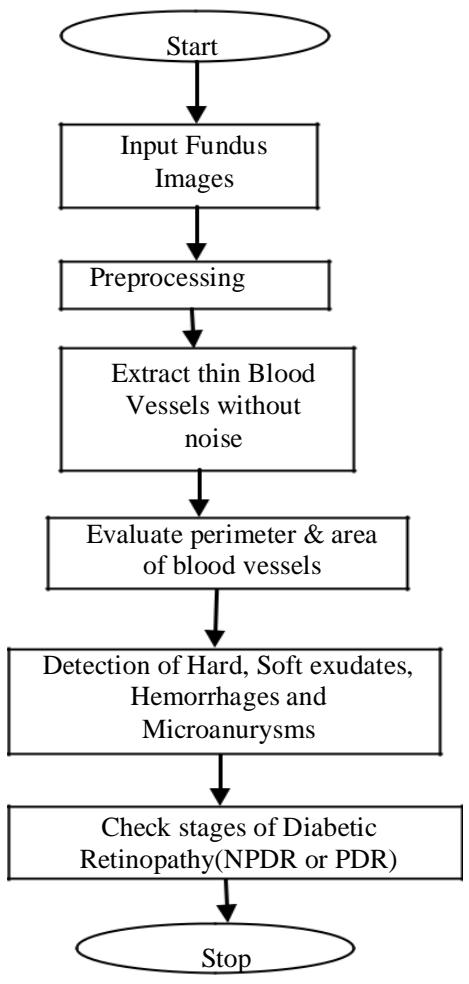

Fig 3: Flow Chart of Proposed Methodology

\section{LIMITATION AND FUTURE SCOPE}

In medical image processing, automatic diagnosis of Diabetic Retinopathy from fundus images has been a dynamic research for an extended time. There are many areas in this field that need improvement such as blood vessel extraction without noise and determination of OD boundary. Therefore, there is not a single technique that can cover all these problems. In this, I proposed a different technique to improve the segmentation by detecting the thin blood vessels without noise more precisely from fundus images with minimum computational difficulties and in less time.

\section{CONCLUSION}

In this Paper, An analysis on detection and categorization of Diabetic Retinopathy(DR) from fundus retinal images has been done. To detect and categorize the type of diabetic retinopathy disorder, some researchers used some classifier and image processing techniques, for identifying the Exudates, Hemorrhages and Microanurysms Morphological processing technique is widely used in most of the research papers. It is significant to extract the retinal blood vessels proficiently so that fundus image can be recognized accurately. The proposed techniques can supplementary improve the line of treatment by diagnosing the small blood vessels more accurately from retinal fundus images with less computational complication and in minimum instance.

\section{REFERENCES}

[1] M. PonnibalaS. Vijayachitra "Extraction of retinal blood vessels and diagnosis of proliferative diabetic retinopathy using extreme learning machine,", Journal of medical imaging and health informatics, vol. 5, pp 248-256, April 2015.

[2] MalavikaBhaskaranand "Automated Diabetic Retinopathy Screening and Monitoring Using Retinal Fundus Image Analysis," vol. 10, pp 254-261, Feb 2016. 
[3] Mr.sagarlachure et al..”Diabetic retinopathy using morphological operations and machine learning," Advanced Computing Conference, June 2015.

[4] SwamidossIssac Niwas, Weisi Lin, Senior Member, IEEE, Chee KeongKwoh, C.-C. Jay Kuo, Fellow, IEEE, Chelvin C. Sng, Maria Cecilia Aquino, and Paul T. K. Chew" Cross-Examination for Angle-Closure Glaucoma Feature Detection,"IEEE Journal of Biomedical and Health Informatics, vol. 20, pp 343354, jan 2015.

[5] ManojKumar S B , Manjunath R, Dr. H S Sheshadri, "Feature extraction from the fundus images for the diagnosis of diabetic retinopathy", International Conference on Emerging Research in Electronics, Computer Science and Technology - 2015.

[6] R. Manjulashree, R Raghupathy Reddy, K.M.M. Rao, "Image Processing for identifying different stages of Diabetic Retinopathy", International Journal of Recent Trends in Engineering \& Technology, Vol 11,June 2014.

[7] N.B. Prakash, G.R. Hemalakshmi, M. Stella Inba Mary, "Automated grading of diabetic retinopathy stages in fundus images using SVM classifier", Journal of Chemical and Pharmaceutical Research, Vol 8, Issue 1, ISSN: 0975-7384, 2016.

[8] Ahmad Taher Azar, Valentina E. Balas, "Classification and Detection of Diabetic Retinopathy" advanced in
Intell. Analysis of Medical Data, SCI 473,pp-135-145, Springer-VerlagBerlin Heidelberg 2013.

[9] M.M. Fraz., P. Remagnino, A. Hoppe, B. Uyyanonvara, "Blood vessel segmentation methodologies in retinal images - A survey", Computer Methods and Programs in Biomedicine pp 127-155, 2012.

[10] Manjiri B Patwari, Ramesh R. Manza, Yogesh M. Rajput "Review on Detection and Classification of Diabetic Retinopathy Lesions Using Image Processing Techniques", International journal of Engineering Research \& Technology, Vol 2 Issue 10 pp 395-399,2013.

[11] Sonam Dilip Solkar, Lekha Das "Survey on Retinal Blood Vessels Segmentation Techniques for Detection of Diabetic Retinopathy", International Journal of Electronics, Electrical and Computional Systems, Vol 6 Issue 6, 2017.

[12] Pavithra, Dr N. Shanthni, "A Survey on Detection of Diabetic Retinopathy and Glaucoma from Fundus Image", International Journal of Science and Research, vol 5 Issue 7 pp 998-1001,July 2017.

[13] Javeria averia Amin, Muhammad Sharif, Mussart Yasmin, "A Review on Recent developments for detection of Diabetic Retinopathy", Hindawi Publishing Corporation Scientifica, 2016. 